\title{
BMJ Open Epidemiology, management and the associated burden of mental health illness, atopic and autoimmune conditions, and common infections in alopecia areata: protocol for an observational study series
}

\author{
Matthew Harries, ${ }^{1}$ Abby E Macbeth, ${ }^{2}$ Susan Holmes, ${ }^{3}$ Andrew R Thompson, ${ }^{4}$ \\ Wing Sin Chiu, ${ }^{5}$ William Romero Gallardo, ${ }^{5}$ Andrew G Messenger, ${ }^{6}$ \\ Christos Tziotzios, ${ }^{7}$ Simon de Lusignan (1) ${ }^{8,9}$
}

To cite: Harries M, Macbeth $\mathrm{AE}$, Holmes S, et al. Epidemiology, management and the associated burden of mental health illness, atopic and autoimmune conditions, and common infections in alopecia areata: protocol for an observational study series. BMJ Open 2021;11:e045718. doi:10.1136/ bmjopen-2020-045718

- Prepublication history and additional supplemental material for this paper are available online. To view these files, please visit the journal online (http://dx.doi.org/10.1136/ bmjopen-2020-045718).

Received 12 0ctober 2020 Accepted 15 0ctober 2021

Check for updates

(C) Author(s) (or their employer(s)) 2021. Re-use permitted under CC BY-NC. No commercial re-use. See rights and permissions. Published by BMJ.

For numbered affiliations see end of article.

Correspondence to

Dr Simon de Lusignan;

simon.delusignan@phc.ox.ac.uk

\section{ABSTRACT}

Introduction Alopecia areata (AA) is a common cause of immune-mediated non-scarring hair loss. Links between $A A$ and common mental health, autoimmune and atopic conditions, and common infections have previously been described but remain incompletely elucidated and contemporary descriptions of the epidemiology of AA in the UK are lacking.

Methods and analysis Retrospective study series using a large population-based cohort (5.2 million) from the 0xford Royal College of General Practitioners (RCGP) Research and Surveillance Centre (RSC) database, exploring four themes: AA epidemiology, mental health comorbidities, autoimmune/atopic associations and common infections. In the epidemiology theme, we will describe the incidence and point prevalence of AA overall and by age, sex and sociodemographic factors. Healthcare utilisation (primary care visits and secondary care referrals) and treatments for AA will also be assessed. In the mental health theme, we will explore the prevalence and incidence of mental health conditions (anxiety, depressive episodes, recurrent depressive disorder, adjustment disorder, agoraphobia, self-harm and parasuicide) in people with AA compared with matched controls. We will also explore the mental health treatment patterns (medication and psychological interventions), time off work and unemployment rates. Within the autoimmune/ atopic associations theme, we will examine the prevalence of atopic (atopic dermatitis, allergic rhinitis, asthma) and autoimmune conditions (Crohn's disease, ulcerative colitis, coeliac disease, type 1 diabetes, Hashimoto's thyroiditis, Graves' disease, rheumatoid arthritis, psoriatic arthritis, ankylosing spondylitis, systemic lupus erythematosus (SLE), polymyalgia rheumatica, Sjögren's syndrome, psoriasis, vitiligo, multiple sclerosis, pernicious anaemia) in people with AA compared with matched controls. We will also estimate the incidence of new-onset atopic and autoimmune conditions after AA diagnosis. Within the common infections theme, we will examine the incidence of common infections (respiratory tract infection,
Strengths and limitations of this study

We will use a nationally representative primary care database of over 5.2 million people to explore the epidemiology of alopecia areata (AA), as well as associations of AA with mental health conditions, autoimmune and atopic conditions, and common infections.

- As national guidelines recommend initial assessment and management of $\mathrm{AA}$ is undertaken in primary care, this study will provide an accurate contemporary analysis of AA epidemiology in England in both children and adults.

- The Oxford Royal College of General Practitioners (RCGP) Research and Surveillance Centre (RSC) primary care data set has a high level of completeness on sociodemographic and geographic data and therefore enables an assessment of variation in the incidence and prevalence of AA across England.

- Oxford RCGP RSC is the primary infectious disease sentinel network for the UK; the common in infections to be explored are all regularly monitored conditions within the network.

- A limitation of the study is that a validated case definition of $A A$ is not available for primary care databases; this will be mitigated by additional analysis to explore the sensitivity of findings when non-specific alopecia diagnostic codes (eg, unspecified alopecia) are included.

pneumonia, acute bronchitis, influenza, skin infection, urinary tract infection, genital infections, gastrointestinal infection, herpes simplex, herpes zoster, meningitis, COVID-19) in people with AA compared with matched controls.

Ethics and dissemination The Health Research Authority decision tool classed this a study of usual practice, ethics approval was not required. Study approval was granted by 
the RCGP RSC Study Approval Committee. Results will be disseminated through peer-reviewed publications.

Observational study registration number NCT04239521.

\section{INTRODUCTION}

Alopecia areata (AA) is a common cause of immunemediated, non-scarring hair loss. ${ }^{1}$ Clinical presentations of AA are heterogeneous, ranging from well-demarcated patches of hair loss on the scalp to total loss of hair on the scalp (alopecia areata totalis) or entire scalp and body (alopecia areata universalis). ${ }^{2}$ Active AA is estimated to affect approximately $0.1 \%-0.2 \%^{2}{ }^{3}$ of the population worldwide, with a lifetime risk of $1.0 \%-2.1 \% .^{4-6}$

Following the initial episode, the majority of those with limited patchy AA will experience spontaneous remission, but over $80 \%$ will have subsequent relapses. ${ }^{7}$ Spontaneous remission has been reported to occur in less than $10 \%$ of those with extensive AA. ${ }^{7}$ A number of treatment options can induce hair growth in people with AA, but none have been shown to have a durable impact on remission. ${ }^{8}$

AA has been associated with a number of autoimmune conditions, supporting the theory of an underlying autoimmune aetiology. ${ }^{9}$ Atopic conditions and mental health disorders have also been demonstrated to be more common in people with $\mathrm{AA},{ }^{9-11}$ although the temporal relationship between psychiatric conditions and AA remains poorly researched. ${ }^{12}$ The association between AA and common infections is not well studied.

This study series using a large contemporary population-based sample aims to explore and describe many important elements of AA over four key areas: (1) the current epidemiology of AA; (2) the prevalence and incidence of psychiatric comorbidities in people with AA; (3) the prevalence and incidence of autoimmune and atopic conditions in people with AA; (4) the incidence of common infections in people with AA.

\section{Current knowledge}

\section{Epidemiology}

Population studies in the USA using the Rochester Epidemiology Project database estimate the lifetime risk of AA to be $2.1 \%$. ${ }^{4}$ The database contained information from all patients registered with a healthcare provider in Olmsted County, Minnesota, from 1990 to 2009. Previous studies using this database from 1971 to 1974 estimated the overall prevalence of AA to be $0.1 \%-0.2 \%,{ }^{13}$ with no published studies reporting prevalence estimates since this. Other epidemiological studies have assessed the incidence of AA in patients referred to dermatology services. Clinicbased studies from around the world have estimated the incidence of AA to be in the region of $0.57 \%-3.8 \% .^{14-18}$

Worldwide, the majority of studies on AA have not found a significant difference in incidence between males and females. ${ }^{5}$ AA onset occurs before the age of 40 in $82 \%-86 \%$ of cases. ${ }^{5}$ To date, there have been no large population-based studies of AA epidemiology in UK populations.
Mental health comorbidities

It is well recognised by clinical experts in AA that the condition can have a profound psychological impact and a possible comorbid burden of mental health conditions. A recent US cross-sectional database study comparing 5605 patients with AA with matched controls found a higher prevalence of mental health diagnoses in the AA group $(32.8 \%$ vs $20.0 \%) .{ }^{19}$ In particular, higher rates of depression (OR 2.18, 95\% CI 1.80 to 2.63 ), anxiety disorders (OR 2.46, 95\% CI 1.91 to 3.16), attention-deficit disorders (OR 8.11, 95\% CI 5.22 to 12.59), adjustment disorders (OR 4.80, 95\% CI 2.58 to 8.91) and obsessive compulsive disorders (OR 4.95, 95\% CI 1.59 to 15.35 ) were observed when compared with controls. ${ }^{19}$ These findings concur with previous work and a similar pattern is seen in other countries. ${ }^{20-23}$

Recently, a British study identified a bidirectional positive association between major depressive disorder and AA. ${ }^{24}$ In a sample of over 400000 participants, major depressive disorder was seen to increase the risk of incident AA by $90 \%$ (HR 1.90, 95\% CI 1.67 to 2.15) and AA was found to increase the risk of incident major depressive disorder by $34 \%$ (HR $1.34,95 \%$ CI 1.23 to 1.46$).^{24}$ The association of other common mental health conditions with AA however was not assessed.

\section{Autoimmune and atopic comorbidities}

A central theory in AA pathogenesis is that it is an autoimmune phenomenon resulting from a disruption of hair follicle 'immune privilege', where numerous immunological mechanisms fail to prevent cytotoxic immune attack on cells and antigens present at that site. ${ }^{25}$

A number of studies have reported autoimmune conditions to occur more frequently in those with AA than in the general population; however, results are conflicting and studies have been limited by potential recruiting, recall and reporting biases. ${ }^{26} \mathrm{~A}$ Taiwanese retrospective cohort study found individuals with AA to have an overall increased risk of developing an autoimmune condition when compared with matched controls (HR 1.86, 95\% CI 1.32 to 2.63$)$. In particular, greater risks of incident SLE (HR 5.02, 95\% CI 2.08 to 12.51), psoriasis (HR 2.01, 95\% CI 1.06 to 3.83 ) and rheumatoid arthritis (RA) (HR $1.79,95 \%$ CI 1.07 to 3.00 ) were observed. ${ }^{27}$ There was no relationship between AA and Hashimoto's thyroiditis or type 1 diabetes (T1DM). A recent systematic review and meta-analysis found AA to be significantly associated with Graves' Disease (OR 2.07, 95\% CI 1.80 to 2.38) and Hashimoto's thyroiditis (OR 2.15, 95\% CI 1.71 to 2.72) but not with SLE, RA, psoriasis or T1DM. ${ }^{9}$

There has also been interest in the prevalence of comorbid atopy in patients with AA. The prevalence of atopy in patients with AA has been reported to be in the range of $11 \%-38.2 \%{ }^{5}$ representing cohorts from the USA, India and Singapore. ${ }^{11} 142829$ A large meta-analysis found patients with AA had higher odds of having atopic dermatitis (OR 2.36, 95\% CI 1.80 to 3.09) or allergic rhinitis (OR 1.33, 95\% CI 1.19 to 1.47 ) when compared with 
controls. ${ }^{9}$ It is not however surprising from a pathogenesis perspective in terms of shared pathways of autoimmune susceptibility; interleukin 13 (IL-13) loci have been associated with autoimmune and atopic conditions, and a genome-wide association study established that IL-13 are also susceptibility loc for AA. ${ }^{30}$

\section{Common infections}

It has been hypothesised that infections may act as triggers for AA; with particular interest in Helicobacter pylori, Cytomegalovirus, Epstein-Barr virus, hepatitis B virus, hepatitis $\mathrm{C}$ virus and HIV, but others have also been suggested. ${ }^{931-38}$ Some case reports have suggested improvement or resolution of AA with treatment of the associated infection. ${ }^{33}{ }^{39}$ However, early small-scale studies have failed to find convincing evidence to support associations. .353640

Multiple genetic loci have been identified to be both protective from infection but increase the risk for autoimmune disease. ${ }^{41}$ Examples include the human leukocyte antigen (HLA) variants HLA-DQ2 and HLA-DQ8 which confer protection against viral infections including influenza but are associated with inflammatory bowel disease, ${ }^{42} 43$ and SH2B3 rs3184504*A gene variant protective against Escherichia coli infection but also detrimental for inflammatory bowel disease. ${ }^{44}$ These loci, which in the past have conferred a genetic advantage, may still confer a reduced infection risk. ${ }^{41}$ It is currently unclear if the genetic predisposition for AA is caused by genetic factors which also confer protection against any common infections. In other autoimmune conditions, infection risk is often increased as a result of the pathological changes resulting from the condition itself, the effects of immunemodulatory treatment or through increased exposure to pathogens through healthcare contact. ${ }^{45-51}$ An understanding of the relative infection rates in people with AA compared with those without is of particular importance with the emergence of Janus kinase inhibitors as a potential treatment option. ${ }^{52}$ While this medication class can produce impressive hair regrowth results even in longstanding AA, their use is associated with some increased infection risk; in particular, urinary tract infections, viral gastroenteritis fungal infections and varicella-zoster infection. ${ }^{52}$

\section{Aims and objectives}

We aim to perform a series of retrospective studies using a large population-based cohort in England over a decade (1 January 2009 to 1 January 2019) exploring AA in four theme areas: epidemiology, mental health, autoimmune and atopic comorbidities, and common infections.

Within the epidemiology theme, our initial objective is to provide a complete description of the incidence and prevalence of AA in England. We will explore the influence of sociodemographic and geographic factors on disease incidence and describe the current service utilisation and management patterns in AA during the year after diagnosis.
Within the mental health theme, we will explore the disease burden of common mental health conditions in people with AA compared with matched controls. We will calculate the prevalence of disease at AA diagnosis, the incidence of disease in the first 2 years after diagnosis and the influence of sociodemographic factors on the prevalence of common mental health conditions in AA. We will describe the mental health treatment burden in people (medication and psychological service use) with AA and depression and anxiety. We will also explore potential associations between $\mathrm{AA}$ and both time off work and unemployment.

Within the autoimmune and atopic comorbidities theme, we will examine the prevalence of atopic and autoimmune conditions at AA diagnosis, and the incidence of new-onset atopic and autoimmune conditions in the first 5 years after diagnosis in people with AA compared with matched controls.

Within the common infections theme, we will examine the incidence of multiple common infections in the first 5 years after diagnosis in people with AA compared with matched controls.

The primary and secondary objectives of each study theme are formally described in the statistical analysis plan (online supplemental additional file 1).

\section{METHODS AND ANALYSIS \\ Data source}

These studies will use routinely collected and collated data from the Oxford Royal College of General Practitioners (RCGP) Research and Surveillance Centre (RSC) database to provide a broadly representative sample of the population of England. The RCGP RSC incorporates pseudonymised primary care records from over 200 general practitioner (GP) practices distributed across England. Details of the cohort included in the database have been published previously. ${ }^{53}$ At the current time, the database contains data from 2.6 million actively registered people and the historic records of up to 5.2 million people over the last 20 years.

The RCGP RSC database includes patient demographics, diagnoses, laboratory test results, primary care issued prescriptions and processes of care such as clinic visits and specialist referrals. These data are coded using the Read code system. UK general practice records provide several advantages for this study type; UK primary care is a registration-based system (a patient can only register with a single GP), records have been computerised since the 1990s and pay-for-performance targets (introduced in 2004 through the quality and outcomes framework) have resulted in consistent high-quality clinical data entry about chronic disease. ${ }^{545}$ Studies in many chronic diseases such as atopic dermatitis, RA, diabetes, inflammatory bowel disease and chronic obstructive pulmonary disease have been published using RCGP RSC data. ${ }^{51}{ }^{56-59}$ RCGP RSC practices get feedback on their data quality, with a particular emphasis on 'episode 
typing'-designating cases first, new or ongoing, which helps differentiate incidence from prevalence. ${ }^{60}$

\section{Study design}

Within RCGP RSC, people with and without AA will be identified using Read diagnostic codes. A range of study designs will be used across the studies and include: repeated cross-sectional design (epidemiology of AA), case-control design (the prevalence of common mental health conditions, atopic and autoimmune conditions, and common infections at AA diagnosis) and matched cohort design (the incidence of common mental health conditions, atopic and autoimmune conditions, and common infections after AA diagnosis).

\section{AA case detection}

We will identify cases of AA using specific diagnostic codes (online supplemental table S1.1, additional file 2). Confirmed AA will be defined as one or more AA codes and no alternative diagnostic code in the subsequent 365 days (alternative diagnoses are defined in online supplemental table S1.1, additional file 2). Specificity of findings to this case definition will be evaluated (see Sensitivity Analysis and online supplemental table S1.2, additional file 2).

\section{Study 1: epidemiology of AA}

The incidence and lifetime prevalence of $A A$

We will describe the incidence and point prevalence of AA over 2009-2018 inclusive, by age group, sex, ethnicity, and socioeconomic status, and geographical region. Ethnicity will be categorised as follows based on UK census categories: White, Asian, Black African/Caribbean, Mixed and Other. ${ }^{61}$ Index of multiple deprivation (IMD) will be used as a measure of socioeconomic status. ${ }^{62}$

\section{Service utilisation in people with AA}

We will assess primary care visit frequency, up to 1 year, in people newly diagnosed with AA compared with matched controls without AA. Measures of healthcare management will also be assessed in people newly diagnosed with AA up to 1 year, comprising: secondary care dermatology referrals (online supplemental table S1.3, additional file 2) and primary care prescriptions for AA treatments (online supplemental table S1.4, additional file 2).

\section{Study 2: mental health conditions in people with AA}

We will assess the prevalence and incidence of three common mental conditions as defined by the International Statistical Classification of Diseases and Related Health Problems 10th Revision classification: depressive episodes (F32), recurrent depressive disorder (F33) and non-phobia-related anxiety disorders (F41). These conditions have been chosen as they represent the most common mental health conditions presenting to primary care. ${ }^{63}$ Conditions will be identified using algorithms validated in UK primary care. We selected the algorithms to be used in the study to optimise the positive predictive value of case identification. In brief, we will use a concurrent diagnosis and treatment code to identify acute depression episodes and for recurrent depressive disorder either a historical code for any depression and current treatment or a recurrent depressive disorder-specific diagnosis code (online supplemental tables S2.1-S2.3, additional file 2). Anxiety disorder will be identified using a concurrent diagnosis of an anxiety episode and treatment code (online supplemental table S2.4, additional file 2).

To assess prevalence, we will perform a case-control analysis to compare the prevalence of mental health conditions between people newly diagnosed with AA and controls. To assess incidence, we will perform a matched cohort analysis in the subset of cases and controls free of the mental health conditions of interest at baseline. In addition, we will examine the prevalence and incidence of other mental health conditions: adjustment disorder, agoraphobia, self-harm and overdose/parasuicide attempts (online supplemental tables S2.5-S2.9, additional file 2).

We will determine the treatment burden of mental health medications in AA (selective serotonin reuptake inhibitors, serotonin and norepinephrine reuptake inhibitors, tricyclic antidepressants, tetracyclic antidepressants, and monoamine oxidase inhibitors, anxiolytic medications) and psychological intervention (referral rates for counselling, secondary care psychiatrist referrals, including the referrals to the clinical psychology services/specialists and UK Improving Access to Psychological Therapies programme (online supplemental table S2.10, additional file 2)).

We will also assess unemployment and time off work in people of working age ${ }^{18-64}$ Unemployment will be identified using codes relating to unemployment recorded in the clinical record or the issuing of IB113 or ESA113 form (online supplemental table S3.1, additional file 2). These forms are completed by GPs when their patients are accessing incapacity benefits (historically) or employment and support allowance (current) because of a disability or health condition affecting their ability to work. Time off work will be assessed by reporting the rates of issue of Med 3 (Statement of Fitness for Work) certification from primary care (online supplemental table S3.2, additional file 2). These 'fit notes' are required by employers when employees have periods of absence from work for sickness lasting longer than 7 days.

\section{Study 3: autoimmune and atopic comorbidities in AA}

We will assess the prevalence and incidence of autoimmune and atopic conditions as listed in table 1 and defined in online supplemental table S4, additional file 2, in people with AA. To assess prevalence, we will perform a case-control study to compare the prevalence of these conditions between cases and controls. To assess incidence, we will perform a matched cohort study to compare the incidence of new autoimmune and atopic conditions in the same cases and controls after the point of matching. Autoimmune and atopic conditions were included if prevalence has been reported to 
Table 1 The autoimmune and atopic conditions to be explored. For full definitions, see online supplemental tables S4.1S4.16, additional file 2

\begin{tabular}{|c|c|}
\hline Disease category & Conditions \\
\hline Atopic conditions & Atopic dermatitis, allergic rhinitis, asthma \\
\hline Gastrointestinal & Crohn's disease ${ }^{\star}$, ulcerative colitis, coeliac disease, pernicious anaemia \\
\hline Rheumatological & $\begin{array}{l}\text { Rheumatoid arthritis, psoriatic arthritis, ankylosing spondylitis, systemic lupus erythematosus, polymyalgia } \\
\text { rheumatica, Sjögren's syndrome }\end{array}$ \\
\hline
\end{tabular}

*While there has been some debate about whether or not Crohn's disease is an autoimmune condition it involves a degree of immune dysregulation and is therefore included for completeness. ${ }^{74}$

be $0.3 \%$ or greater. ${ }^{94-67}$ This cut-off was based on power calculations suggesting this is minimum background prevalence required to detect a twofold difference in prevalence (online supplemental additional file 1). Excluded autoimmune conditions due to low prevalence are Addison's disease, autoimmune haemolytic anaemia, immune thrombocytopenic purpura, autoimmune hepatitis, systemic sclerosis, myasthenia gravis and systemic vasculitides.

\section{Study 4: common infections in AA}

We will assess the incidence of common infections in people with AA using a matched cohort study to compare the incidence of common infections in cases and controls. The common infections to be examined are listed in table 2 and defined in online supplemental table S5, additional file 2.

\section{Statistical methods}

The full statistical analysis plan is provided in online supplemental additional file 1. Briefly, for study 1, AA prevalence will be calculated by dividing the number of AA cases by the total number of eligible people in the study population. Point prevalence will be reported for the cohort overall and stratified by sex and age. AA incidence will be calculated by dividing the total number of new AA cases by the total person-years of follow-up. Incidence rates will be stratified by calendar year, sociodemographic factors (age group, sex, ethnicity, rural/urban classification and socioeconomic status) and geographical region. Adjusted incidence rates will be estimated using Poisson regression.

Service utilisation (studies 1 and 2) will be evaluated by calculation of annual primary care attendance rates,

Table 2 The common infections to be explored

\begin{tabular}{ll}
\hline Infection & Description \\
\hline Upper respiratory tract infection & $\begin{array}{l}\text { All infections of the upper respiratory tract including rhinitis, pharyngitis, sinusitis and the } \\
\text { common cold }\end{array}$ \\
\hline Lower respiratory tract infection & All infections of the lower respiratory tract including pneumonia and acute bronchitis \\
\hline Pneumonia & A subgroup of LRTI consisting of bacterial and viral pneumonias \\
\hline Acute bronchitis & $\begin{array}{l}\text { A subgroup of LRTI consisting of inflammation of the large and medium airways usually of } \\
\text { viral origin }\end{array}$ \\
\hline Influenza & Confirmed cases of influenza and cases of influenza-like illness \\
\hline Skin infection & Cellulitis and other infective skin conditions \\
\hline Urinary tract infection & Infections of the urinary tract including cystitis and pyelonephritis \\
\hline Genital infections & All infections of the genital tract including sexually transmitted infections and non-sexually \\
transmitted infections
\end{tabular}

*Inclusion of COVID-19 will depend on data availability.

LRTI, lower respiratory tract infection. 
treatment rates (prescriptions per person per year) and the total proportion of people with one or more referrals for additional services (dermatology review (study 1), psychiatrist referral and counselling (study 2)).

For studies 1 (for comparison of primary care visit frequency only), 2 and 3, matching will be performed as follows: control cohorts will be defined by matching cases with people without a diagnosis of AA or other alopecia. The follow-up period for each matched control will begin at the start of follow-up of their matched case. Each case will be nearest neighbour matched with four controls by age and sex within GP practices. After matching, if there are substantial differences in socioeconomic status between cases and controls, rematching with IMD as an additional matching criterion will be considered. Study 4 will use the same nearest neighbour matching process but cases will be defined as people diagnosed with AA on or before 1 January 2014, people who develop AA during follow-up will be excluded from the pool of available controls prior to matching.

For study 2, we will report the prevalence of mental health conditions by sociodemographic subgroups in cases and controls. The burden of treatment for mental health conditions will be estimated as the total proportion of people prescribed each treatment within 1 year after the study start. The burden of sick days will be estimated as the total proportion of people issued a Med 3 certificate. To assess differences in incident mental health conditions in the 2 years after the study start, we will use adjusted Cox regression stratified by case/control status. Study 3 (atopic and autoimmune conditions) will similarly compare baseline disease prevalence in cases and controls, and compare incidence of new-onset conditions in the 5 years after the study start using adjusted Cox regression.

Study 4 will compare infection incidence in cases and controls up to 5 years. Infection incidence rates will be estimated by dividing the number of incident common infections by total person-years of follow-up. Adjusted Poisson regression will be used to estimate the association between AA and rates of common infection.

\section{Sensitivity analyses}

To evaluate the specificity of any identified associations to our AA case definition, in sensitivity analysis we will extract alopecia recorded where the type has not been specified (online supplemental table S1.1, additional file 2). We will then assess the characteristics of people with non-specific alopecia codes only (ie, no prior or subsequent codes specifying alopecia type) compared with the primary AA cohort. If the characteristics are not markedly different, which would indicate the groups are clinically different and not comparable, we will evaluate major study outcomes in this non-specific AA cohort and compare results to those obtained for the primary AA cohort. We will also evaluate whether stratifying analyses by alopecia extent is possible, dependent on how well this is recorded in primary care data (online supplemental table S1.2, additional file 2).

In study 4, we will use a Cox regression to estimate time to first infection. We will also compare the results of the study outcomes when (1) people who develop AA during the follow-up are included as cases and (2) when we perform matching using the time of AA diagnosis and follow for up to 5 years after diagnosis (or matched date for controls).

\section{DISCUSSION}

\section{Clinical implications}

Results derived from this study will be useful in monitoring trends in the incidence of AA in England and may identify factors that influence the disease distribution and treatment patterns. Furthermore, obtaining an accurate picture of the state of AA in the UK will help inform health needs assessments and policy development.

A greater understanding of the prevalence of mental health conditions associated with AA and the directionality of these relationships may help with the development of psychological interventions in AA, as the approach required would likely differ if the symptoms patients are experiencing are pervasive or reactive to an episode of hair loss. ${ }^{1268}$

Awareness of AA as a potential risk factor or associated comorbidity for certain autoimmune and atopic conditions may help reduce delays in diagnosis for these conditions and increase vigilance of GPs and dermatologists when reviewing their patients with AA in detecting coexistent conditions. Future work may focus on whether improved control of an underlying autoimmune or atopic condition may improve chances of achieving remission in AA.

Finally, analysing associations between AA and common infections may provide evidence to support the prescribing of immune-modulating medications for the treatment of AA.

\section{Strengths and limitations of the research methods}

Several strengths of our study design are noteworthy. Only two previous studies on the incidence and prevalence of AA have been population based, with the vast majority being conducted on clinic patients and thus leading to skewed prevalence estimates. This study will also provide a contemporary picture of the burden of $\mathrm{AA}$, as the previous population-based studies were over 20 years ago, and this will be the first AA epidemiology study conducted in the UK. To our knowledge, our study examining common infections will be the first study to attempt to answer the question of whether AA confers protection or increased risk for common infections. A final key strength of this study is the use of the national infections surveillance network to analyse infection risk; with regular feedback to practices with a focus on the quality of infections recording the quality of recording of 
these infection events higher in this network than other data sources.

A limitation of the study is the lack of a validated AA case definition using primary care data. We are unable to develop one as we have access only to coded anonymised healthcare record data for this study. However, our ontological approach to detecting cases of AA and associated comorbidities will improve accuracy compared with the use of diagnostic codes alone. ${ }^{69} 70$ Exclusion of individuals with potentially confounding conditions (ie, other causes of hair loss) will also improve the accuracy of our estimates. The algorithms used to detect common mental health conditions have been previously validated in UK primary care. The completeness of GP data coding for prescriptions, referrals and issue of Med 3 certificates will ensure that our findings on AA and comorbidity management are robust and reliable, and the 'episode typing' functionality of the RCGP RSC database will ensure incident and prevalent cases are appropriately assigned. The RCGP RSC database has been shown to be a representative sample of the UK population, ${ }^{53}$ and hence our results are broadly generalisable to the UK as a whole but should not be extended to populations which are dissimilar.

There are also other several limitations of the study design that are worthy of consideration. While a previous study has shown the ethnic breakdown in primary care data is similar to the UK census and suitable for research, ${ }^{71}$ $86 \%$ of people in the UK are from white ethnic groups, limiting power to evaluate people of non-white ethnicity despite the large sample size. Findings may not be generalisable to countries with a substantially different ethnicity mix to the UK. This study does not attempt to detect cases of AA (or the comorbidities of interest) that do not present to medical attention and hence may underestimate the true burden of disease. Furthermore, only coded data in the database can be used and hence we rely on accurate coding. Some individuals may need to be excluded due to incomplete data, although in similar studies the exclusion rate due to incomplete data has been low. ${ }^{72} 73$

Prevalence of Med 3 certification is well captured in primary care but the reason for issue is not available, meaning that we cannot identify the condition relating to the Med 3 certification. We cannot therefore determine whether the sick leave was as a result of AA. However, our case-control approach will provide a method for comparing Med 3 certificate issue rates in those affected and unaffected by AA. The use of Read codes to identify unemployment is likely to systemically underestimate unemployment prevalence but is likely to be suitable for providing a comparison with controls.

Another limitation is the possibility of missing treatment information from secondary care. Secondary care records in the UK are frequently not computerised and prescriptions are still commonly handwritten. At present, there is no mechanism for universally capturing secondary care prescriptions and linking them to primary care records. We are therefore likely to underestimate prescription rates for those with the most severe disease who are seen frequently in secondary care. Where prescriptions are issued from primary care the indication for the prescription is not available and we can therefore not confirm that the prescription is for the treatment of AA.

\section{Data management}

Individual patient data will be anonymised at the point of data extraction. All data will remain in anonymised form and will be held on a secure server at the University of Surrey. All statistical analyses will be performed using R software.

\section{Patient and public involvement}

The design of this protocol did not involve patients or this public.

\section{Ethics approval and dissemination}

Study approval has been granted by the RCGP RSC Study Approval Committee. The study does not require formal research ethics committee approval as it will use anonymised routinely collected healthcare data; as defined by the National Health Service Health Research Authority research decision tool (http://www.hra-decisiontools. org.uk/research/) and the University of Surrey's own Self-Assessment for Governance and Ethics tool. This observational study has been registered with ClinicalTrials.gov (NCT04239521). The data will not be used for any purposes other than for the research which is described in the respective protocols and which has been approved by the RCGP RSC Study Approval Committee. We plan to publish the final study results in peer-reviewed journals and present early findings at scientific meetings.

\section{CONCLUSIONS}

This study series aims to provide the first large-scale population-based analysis of the epidemiology of AA in the UK and the current patterns of healthcare utilisation and treatments in this group. We also aim to provide an extensive assessment of the association between common mental health conditions and work-related difficulties in people with AA; reporting the prevalence and incidence of these conditions in people with new-onset AA and matched controls. We aim to provide an extensive evaluation of the prevalence and incidence of autoimmune and atopic conditions in people with new-onset AA and matched controls. Finally, we aim to provide evidence for baseline common infection risk in people with AA which can be used to support treatment decisions regarding the use of immune-modulating medications for AA. This study series will extend our knowledge of the current disease burden from AA and associated conditions.-

\section{Author affiliations}

${ }^{1}$ The Dermatology Centre, Salford Royal Hospital, Northern Care Alliance NHS Foundation Trust, Greater Manchester, UK

${ }^{2}$ Department of Dermatology, Norfolk and Norwich University Hospitals NHS Foundation Trust, Norwich, UK 
${ }^{3}$ Alan Lyell Centre for Dermatology, Queen Elizabeth University Hospital, Glasgow, UK

${ }^{4}$ South Wales Clinical Psychology Training Programme, Department of Psychology, Cardiff University, Cardiff, UK

${ }^{5}$ Pfizer, Tadworth, UK

${ }^{6}$ Department of Dermatology, Royal Hallamshire Hospital, Sheffield, UK

${ }^{7}$ St John's Institute of Dermatology, King's College London, London, UK

${ }^{8}$ Nuffield Department of Primary Care Health Sciences, University of Oxford, Oxford, UK

${ }^{9}$ Department of Clinical and Experimental Medicine, University of Surrey, Guildford, UK

\section{Twitter Simon de Lusignan @Lusignan_S}

Acknowledgements Medical writing and editorial support was provided by Louise Jordan, Anita Lynam, Andrew McGovern and John Dennis at Momentum Data and was funded by Pfizer. The authors would like to acknowledge the project management support from Filipa Ferreira of the University of Oxford and University of Surrey. MH was supported by the NIHR Manchester Biomedical Research Centre.

Contributors The study was designed and developed by CT, MH, SH, ART, AEM, WSC, WRG and AGM with input from SdL. The protocol writing was directed and critically reviewed by CT, MH, SH, ART, AEM, WSC, WRG, SdL and AGM.

Funding This research was sponsored and funded by Pfizer.

Competing interests SdL is Director of RCGP RSC. He has received funding for projects from Eli Lilly, AstraZeneca, GSK, Seqirus and Takeda—all through his universities and none related to this study. WSC and WRG are employees of Pfizer. $\mathrm{SH}$ and $\mathrm{CT}$ are investigators on the Pfizer-funded Allegro clinical trial.

Patient consent for publication Not applicable.

Provenance and peer review Not commissioned; externally peer reviewed.

Supplemental material This content has been supplied by the author(s). It has not been vetted by BMJ Publishing Group Limited (BMJ) and may not have been peer-reviewed. Any opinions or recommendations discussed are solely those of the author(s) and are not endorsed by BMJ. BMJ disclaims all liability and responsibility arising from any reliance placed on the content. Where the content includes any translated material, BMJ does not warrant the accuracy and reliability of the translations (including but not limited to local regulations, clinical guidelines, terminology, drug names and drug dosages), and is not responsible for any error and/or omissions arising from translation and adaptation or otherwise.

Open access This is an open access article distributed in accordance with the Creative Commons Attribution Non Commercial (CC BY-NC 4.0) license, which permits others to distribute, remix, adapt, build upon this work non-commercially, and license their derivative works on different terms, provided the original work is properly cited, appropriate credit is given, any changes made indicated, and the use is non-commercial. See: http://creativecommons.org/licenses/by-nc/4.0/.

ORCID iD

Simon de Lusignan http://orcid.org/0000-0001-5613-6810

\section{REFERENCES}

1 Hordinsky MK. Overview of alopecia areata. J Investig Dermatol Symp Proc 2013;16:S13-15.

2 Alkhalifah A, Alsantali A, Wang E, et al. Alopecia areata update: Part I. clinical picture, histopathology, and pathogenesis. J Am Acad Dermatol 2010;62:177-88, quiz 189-90.

3 Madani S, Shapiro J. Alopecia areata update. J Am Acad Dermatol 2000;42:549-66; quiz 567-70.

4 Mirzoyev SA, Schrum AG, Davis MDP, et al. Lifetime incidence risk of alopecia areata estimated at $2.1 \%$ by Rochester Epidemiology Project, 1990-2009. J Invest Dermatol 2014;134:1141-2.

5 Villasante Fricke AC, Miteva M. Epidemiology and burden of alopecia areata: a systematic review. Clin Cosmet Investig Dermatol 2015;8:397-403.

6 Richard M-A, Corgibet F, Beylot-Barry M, et al. Sex- and ageadjusted prevalence estimates of five chronic inflammatory skin diseases in France: results of the "OBJECTIFS Peau » study. J Eur Acad Dermatol Venereol 2018;32:1967-71.

7 Harries MJ, Sun J, Paus R, et al. Management of alopecia areata. BMJ 2010;341:c3671.

8 Cochrane. Treatments for alopecia aerate, alopecia totalis and alopecia universalis, 2008.
9 Lee $\mathrm{S}$, Lee $\mathrm{H}$, Lee $\mathrm{CH}$, et al. Comorbidities in alopecia areata: a systematic review and meta-analysis. J Am Acad Dermatol 2019;80:466-77.

10 Montgomery K, White C, Thompson A. A mixed methods survey of social anxiety, anxiety, depression and Wig use in alopecia. BMJ Open 2017;7:e015468.

11 Huang KP, Mullangi S, Guo Y, et al. Autoimmune, atopic, and mental health comorbid conditions associated with alopecia areata in the United States. JAMA Dermatol 2013;149:789-94.

12 Hunt N, McHale S. The psychological impact of alopecia. BMJ 2005;331:951-3.

13 Safavi K. Prevalence of alopecia areata in the first National health and nutrition examination survey. Arch Dermatol 1992;128:702.

14 Sharma VK, Dawn G, Kumar B. Profile of alopecia areata in northern India. Int J Dermatol 1996;35:22-7.

15 Tan E, Tay Y-K, Goh C-L, et al. The pattern and profile of alopecia areata in Singapore--a study of 219 Asians. Int J Dermatol 2002;41:748-53.

16 Yang S, Yang J, Liu JB, et al. The genetic epidemiology of alopecia areata in China. Br J Dermatol 2004;151:16-23.

17 Guzmán-Sánchez DA, Villanueva-Quintero GD, Alfaro Alfaro N, et al. A clinical study of alopecia areata in Mexico. Int J Dermatol 2007;46:1308-10.

18 Furue M, Yamazaki S, Jimbow K, et al. Prevalence of dermatological disorders in Japan: a nationwide, cross-sectional, seasonal, multicenter, hospital-based study. J Dermatol 2011;38:310-20.

19 Singam V, Patel KR, Lee HH, et al. Association of alopecia areata with hospitalization for mental health disorders in US adults. J Am Acad Dermatol 2019;80:792-4.

20 Sellami R, Masmoudi J, Ouali U, et al. The relationship between alopecia areata and alexithymia, anxiety and depression: a casecontrol study. Indian J Dermatol 2014;59:421.

21 Ruiz-Doblado S, Carrizosa A, García-Hernández MJ. Alopecia areata: psychiatric comorbidity and adjustment to illness. Int J Dermatol 2003;42:434-7.

22 Baghestani S, Zare S, Seddigh SH. Severity of depression and anxiety in patients with alopecia areata in Bandar Abbas, Iran. Dermatol Reports 2015;7:6063.

23 Chu S-Y, Chen Y-J, Tseng W-C, et al. Psychiatric comorbidities in patients with alopecia areata in Taiwan: a case-control study. $\mathrm{Br} \mathrm{J}$ Dermatol 2012;166:525-31.

24 Vallerand IA, Lewinson RT, Parsons LM, et al. Assessment of a bidirectional association between major depressive disorder and alopecia areata. JAMA Dermatol 2019;155:475.

25 Paus R, Ito N, Takigawa M, et al. The hair follicle and immune privilege. J Investig Dermatol Symp Proc 2003;8:188-94.

26 Barahmani N, Schabath MB, Duvic M, et al. History of atopy or autoimmunity increases risk of alopecia areata. J Am Acad Dermatol 2009;61:581-91.

27 Chen $\mathrm{C}-\mathrm{H}$, Wang $\mathrm{K}-\mathrm{H}$, Lin $\mathrm{H}-\mathrm{C}$, et al. Follow-Up study on the relationship between alopecia areata and risk of autoimmune diseases. J Dermatol 2016;43:228-9.

28 Tosti A. Practice gaps. alopecia areata and comorbid conditions. JAMA Dermatol 2013;149:794.

29 Tan E, Tay Y-K, Giam Y-C. A clinical study of childhood alopecia areata in Singapore. Pediatr Dermatol 2002;19:298-301.

30 Jagielska D, Redler S, Brockschmidt FF, et al. Follow-up study of the first genome-wide association scan in alopecia areata: IL13 and KIAA0350 as susceptibility loci supported with genome-wide significance. J Invest Dermatol 2012;132:2192-7.

31 Bhardwaj EK, Trüeb RM. Acute diffuse and total alopecia of the female scalp associated with borrelia-infection. Int J Trichology 2015;7:26-8.

32 Cho M, Cohen PR, Duvic M. Vitiligo and alopecia areata in patients with human immunodeficiency virus infection. South Med J 1995;88:489-91.

33 Gil Montoya JA, Cutando Soriano A, Jimenez Prat J. Alopecia areata of dental origin. Med Oral 2002;7:303-8.

34 Ito T, Tokura Y. Alopecia areata triggered or exacerbated by swine flu virus infection. J Dermatol 2012;39:863-4.

35 Jackow C, Puffer N, Hordinsky M, et al. Alopecia areata and cytomegalovirus infection in twins: genes versus environment? $\mathrm{J} \mathrm{Am}$ Acad Dermatol 1998;38:418-25.

36 OffidaniA, Amerio P, Bernardini ML, et al. Role of cytomegalovirus replication in alopecia areata pathogenesis. J Cutan Med Surg 2000;4:63-5.

37 Paoletti V, Mammarella A, Basili S, et al. Prevalence and clinical features of skin diseases in chronic HCV infection. A prospective study in 96 patients. Panminerva Med 2002;44:349-52. 
38 Richardson CT, Hayden MS, Gilmore ES, et al. Evaluation of the relationship between alopecia areata and viral antigen exposure. Am J Clin Dermatol 2018;19:119-26.

39 Kartal ED, Alpat SN, Ozgunes I, et al. Reversible alopecia universalis secondary to PEG-interferon alpha-2b and ribavirin combination therapy in a patient with chronic hepatitis $\mathrm{C}$ virus infection. Eur $\mathrm{J}$ Gastroenterol Hepatol 2007:19:817-20.

40 Abdel Hafez HZ, Mahran AM, Hofny EM, et al. Alopecia areata is not associated with Helicobacter pylori. Indian J Dermatol 2009;54:17-19.

41 Domínguez-Andrés J, Netea MG. Impact of historic migrations and evolutionary processes on human immunity. Trends Immunol 2019;40:1105-19.

42 Dubois PCA, Trynka G, Franke L, et al. Multiple common variants for celiac disease influencing immune gene expression. Nat Genet 2010;42:295-302.

43 Hung S-C, Hou T, Jiang W, et al. Epitope selection for HLA-DQ2 presentation: implications for celiac disease and viral defense. $J$ Immunol 2019;202:2558-69.

44 Zhernakova A, Elbers CC, Ferwerda B, et al. Evolutionary and functional analysis of celiac risk loci reveals $\mathrm{SH} 2 \mathrm{~B} 3$ as a protective factor against bacterial infection. Am J Hum Genet 2010;86:970-7.

45 Kucharzik T, Maaser C. Infections and chronic inflammatory bowel disease. Viszeralmedizin 2014;30:326-32.

46 Toruner M, Loftus EV, Harmsen WS, et al. Risk factors for opportunistic infections in patients with inflammatory bowel disease. Gastroenterology 2008;134:929-36.

47 Brassard P, Bitton A, Suissa A, et al. Oral corticosteroids and the risk of serious infections in patients with elderly-onset inflammatory bowel diseases. Am J Gastroenterol 2014;109:109.

48 Lichtenstein GR, Feagan BG, Cohen RD, et al. Serious infections and mortality in association with therapies for Crohn's disease: treat registry. Clin Gastroenterol Hepatol 2006;4:621-30.

49 Lichtenstein GR, Feagan BG, Cohen RD, et al. Serious infection and mortality in patients with Crohn's disease: more than 5 years of follow-up in the TREAT ${ }^{\text {TM }}$ registry. Am J Gastroenterol 2012;107:1409-22.

50 Fardet L, Petersen I, Nazareth I. Common infections in patients prescribed systemic glucocorticoids in primary care: a populationbased cohort study. PLoS Med 2016;13:e1002024.

51 Nikiphorou E, de Lusignan S, Mallen C. Haematological abnormalities in new-onset rheumatoid arthritis and risk of common infections: a population-based study. Rheumatology 2019;59.

52 Gilhar A, Keren A, Paus R. Jak inhibitors and alopecia areata. Lancet 2019;393:318-9.

53 Correa A, Hinton W, McGovern A, et al. Royal College of general practitioners research and surveillance centre (RCGP RSC) sentinel network: a cohort profile. BMJ Open 2016;6:e011092.

54 de Lusignan S, van Weel C. The use of routinely collected computer data for research in primary care: opportunities and challenges. Fam Pract 2006;23:253-63.

55 de Lusignan S, Metsemakers JF, Houwink P, et al. Routinely collected general practice data: goldmines for research? A report of the European Federation for medical informatics primary care informatics Working Group (EFMI PCIWG) from MIE2006, Maastricht, the Netherlands. Inform Prim Care 2006;14:203-9.

56 Woodmansey C, McGovern AP, McCullough KA, et al. Incidence, demographics, and clinical characteristics of diabetes of the exocrine pancreas (type 3C): a retrospective cohort study. Diabetes Care 2017;40:1486-93.

57 Nikiphorou E, de Lusignan S, Mallen C, et al. Prognostic value of comorbidity indices and lung diseases in early rheumatoid arthritis: a UK population-based study. Rheumatology 2020;59:1296-305.

58 de Lusignan S, Alexander H, Broderick C, et al. Epidemiology and management of atopic dermatitis in England: an observational cohort study protocol. BMJ Open 2020;10:e037518.

59 Galloway J, Barrett K, Irving P, et al. Risk of venous thromboembolism in immune-mediated inflammatory diseases: a UK matched cohort study. RMD Open 2020;6:e001392.

60 Pathirannehelage S, Kumarapeli P, Byford R, et al. Uptake of a Dashboard designed to give RealTime feedback to a sentinel network about key data required for influenza vaccine effectiveness studies. Stud Health Technol Inform 2018;247:161-5.

61 Office for National Statistics. Ethnicity, 2018. Available: https://www. ons.gov.uk/peoplepopulationandcommunity/culturalidentity/ethnicity [Accessed 2 Jan 2020].

62 Ministry of Housing CLG. English indices of deprivation 2015: technical report, 2015. Available: https://www.gov.uk/government/ publications/english-indices-of-deprivation-2015-technical-report [Accessed 2 Jan 2020].

63 Kessler RC, Chiu WT, Demler O, et al. Prevalence, severity, and comorbidity of 12-month DSM-IV disorders in the National comorbidity survey replication. Arch Gen Psychiatry 2005;62:617-27.

64 Hayter SM, Cook MC. Updated assessment of the prevalence, spectrum and case definition of autoimmune disease. Autoimmun Rev 2012;11:754-65.

65 Cooper GS, Bynum MLK, Somers EC. Recent insights in the epidemiology of autoimmune diseases: improved prevalence estimates and understanding of clustering of diseases. J Autoimmun 2009;33:197-207.

66 Dean LE, Jones GT, MacDonald AG, et al. Global prevalence of ankylosing spondylitis. Rheumatology 2014;53:650-7.

67 Gladman DD, Antoni C, Mease P, et al. Psoriatic arthritis: epidemiology, clinical features, course, and outcome. Ann Rheum Dis 2005;64 Suppl 2:ii14-7.

68 Prickitt J, McMichael AJ, Gallagher L, et al. Helping patients cope with chronic alopecia areata. Dermatol Nurs 2004;16:237-41.

69 Rayner L, Sherlock J, Creagh-Brown B, et al. The prevalence of COPD in England: an ontological approach to case detection in primary care. Respir Med 2017;132:217-25.

70 De Lusignan S. In this issue: ontologies a key concept in informatics and key for open definitions of cases, exposures, and outcome measures. J Innov Health Inform 2015;22.

71 Mathur R, Bhaskaran K, Chaturvedi N, et al. Completeness and usability of ethnicity data in UK-based primary care and hospital databases. J Public Health 2014;36:684-92.

72 Rayner LH, Mcgovern A, Sherlock J, et al. The impact of therapy on the risk of asthma in type 2 diabetes. Clin Respir J 2019;13:299-305.

73 Kumar S, de Lusignan S, McGovern A, et al. Ischaemic stroke, haemorrhage, and mortality in older patients with chronic kidney disease newly started on anticoagulation for atrial fibrillation: a population based study from UK primary care. BMJ 2018;360:k342.

74 Behr MA, Divangahi M, Lalande J-D. What's in a name? The (mis)labelling of Crohn's as an autoimmune disease. Lancet 2010;376:202-3. 